DOI: 10.12957/demetra.2017.25465

\title{
Composição centesimal, compostos bioativos e parâmetros físico-químicos da mama-cadela (Brosimum gaudichaudii Tréc) proveniente do Cerrado Mineiro
}

\section{Proximate composition, bioactive compounds and physicochemical parameters in mama- cadela (Brosimum gaudichaudii Tréc) from Cerrado Mineiro}

Lucas Rogério Braz Land'

Florença Maria Borges ${ }^{1}$

Danielle Oliveira Borges ${ }^{1}$

Grazieli Benedetti Pascoal ${ }^{1}$

1 Universidade Federal de Uberlândia, Faculdade de Medicina, Curso de Nutrição. Uberlândia-MG, Brasil.

Correspondência / Correspondence E-mail:grazi_nutri@yahoo.com.br

\section{Resumo}

Introdução: O Cerrado apresenta grande diversidade de frutos nativos, muitos dos quais comestíveis e considerados fontes de nutrientes, com destaque para a mama-cadela (Brosimum gaudichaudii Tréc). Objetivo: Analisar a composição centesimal, alguns compostos bioativos e parâmetros físico-químicos da mama-cadela. Metodologia: A mama-cadela foi adquirida por um fornecedor de Uberlândia (MG), as análises laboratoriais foram feitas segundo metodologias propostas pela Association of Official Analytical Chemists e pelo Instituto Adolfo Lutz e em triplicata, obtendo-se as médias e desvios-padrão. Resultados: a mama-cadela apresentou (na base úmida): 77,63\% umidade; 1,63\% proteínas; 0,60\% lipídios; $0,82 \%$ cinzas; $13,35 \%$ carboidratos "disponíveis"; 5,11\% fibras alimentares; $5,96 \mathrm{pH} ; 5,85 \%$ acidez; $28,85 \%$ atividade antioxidante; $14,92 \mathrm{mg} \%$ vitamina C; e 46,47 mgEAG\% compostos fenólicos. Conclusão: A mama-cadela se destacou nos teores de fibras alimentares e compostos fenólicos. Ela pode ser inserida na alimentação de populações do Cerrado, às quais possuem acesso a este fruto, contribuindo para uma alimentação saudável e garantindo a segurança alimentar e nutricional.

Palavras-chave: Cerrado. Fruto. Alimento Funcional. Compostos Fenólicos.

\section{Abstract}

Introduction: The Cerrado presents a large diversity of native fruits, many of which are edible and considered sources of nutrients, especially mama-cadela (Brosimum gaudichaudii Trec). 
Aim: To analyze the chemical composition, some bioactive compounds and physicochemical parameters in mama-cadela. Methodology: Mama-cadela was obtained by a provider located in Uberlândia (MG), analysis were made according to methodologies proposed by the Association of Official Analytical Chemists and Adolfo Lutz Institute. Analyses were performed in triplicate and means and standard deviations were obtained. Results: Mamacadela presented (wet weight): $77.63 \%$ moisture; $1.63 \%$ protein; $0.60 \%$ lipids; $0.82 \%$ ash; $13.35 \%$ "available" carbohydrates; $5.11 \%$ dietary fiber; pH 5.96; $5.85 \%$ acidity; $28.85 \%$ antioxidant activity; $14.92 \mathrm{mg} \%$ vitamin C; and $46.47 \mathrm{mgGAE} \%$ phenolic compounds. Conclusion: Mama-cadela was highlighted in dietary fiber and phenolic compounds. It can be inserted into the diet of the Cerrado populations, which have access to this type of fruit, contributing to a healthy diet and ensuring food and nutrition security.

Keywords: Cerrado. Fruit. Functional Food. Phenolic Compounds.

\section{Introdução}

Na América do Sul, mais especificamente no Brasil, encontra-se um dos biomas mais importantes do mundo: o Cerrado. ${ }^{1}$ O Cerrado brasileiro ocupa pouco mais de 2 milhões de $\mathrm{km}^{2}$ (cerca de 22\% do território), abrangendo dez estados (Goiás, Minas Gerais, Rondônia, Mato Grosso, Mato Grosso do Sul, Bahia, Tocantins, Maranhão, Piauí, Pará) e o Distrito Federal.2,3

O Cerrado apresenta uma fauna e flora bastante peculiar e a presença de inúmeros lençóis freáticos, que dão origem a bacias hidrográficas, justifica a necessidade de preservação desse bioma, visto que nas últimas décadas tem-se observado que a agropecuária mecanizada vem sofrendo grande expansão, além das queimadas, provocando um processo de degradação da natureza. ${ }^{3,4}$ Por outro lado, a agroindústria, voltada para o mercado dos frutos do Cerrado, tem crescido, favorecendo o desenvolvimento da população e da região, onde são cultivados esses frutos. ${ }^{5}$

No geral, o Cerrado apresenta uma grande diversidade de frutos nativos, muitos dos quais são comestíveis e considerados fontes de nutrientes, como: proteínas, carboidratos "disponíveis", fibras alimentares, lipídios, vitaminas, minerais e inúmeros compostos bioativos (destaque para os compostos fenólicos e os carotenoides). Os compostos bioativos encontrados nesses frutos estão associados à diminuição do risco de desenvolvimento de diversas doenças crônicas não transmissíveis (obesidade, diabetes mellitus, câncer e doenças cardiovasculares). 1,2,6 
Os frutos do Cerrado têm sido utilizados pela indústria alimentícia, farmacêutica e cosmética, gerando diversos produtos importantes para a sociedade. Além disso, o cultivo dos frutos do Cerrado gera renda a pequenos e médios produtores locais, garantindo a segurança alimentar e nutricional de suas famílias e retardando o desmatamento e o crescimento desordenado de grandes lavouras. Entre os produtos caseiros com frutos do Cerrado que podem ser elaborados pelos produtores locais estão: geleias, doces de compota, licores, sorvetes, picolés e conservas. Um dado interessante é que os frutos do Cerrado fazem parte da cultura alimentar da região, estando presente em diversas preparações, como: arroz com pequi, torta de jatobá, sorvete de mangaba e outros sabores, licor de cagaita e a paçoca de buriti. ${ }^{7}$

Entre as várias espécies de frutos do Cerrado, pode-se destacar a mama-cadela (Brosimum Gaudichaudii Tréc). ${ }^{8}$ A mama-cadela, conhecida também por algodãozinho, bureré, amoreirado-mato, mamica-de-cachorra, mamica-de-cadela ou marjejum, apresenta uma parte comestível carnosa e a madeira da sua árvore é comumente utilizada na fabricação de móveis. Outras partes da mama-cadela (cascas, raízes e folhas) são utilizadas também na medicina, principalmente no tratamento de vitiligo, cujos princípios ativos são as fucumarinas, o bergapteno e o psoraleno. ${ }^{9}$ Por outro lado, a mama-cadela também é importante para a alimentação da população do Cerrado, na qual é misturada à farinha de mandioca, formando uma pasta consumida como complemento alimentar. Em adição, a mama-cadela também apresenta um sabor doce e textura semelhante à goma de mascar, sendo muito apreciada pelas crianças..$^{10}$

Com base no exposto, o presente estudo objetivou analisar as características nutricionais (umidade, carboidratos “disponíveis", fibras alimentares, proteínas, lipídios e cinzas), físico-químicas ( $\mathrm{pH}$, acidez total titulável e atividade antioxidante) e determinação de alguns compostos bioativos, com potente ação antioxidante, como os compostos fenólicos e a vitamina C) na mama-cadela (Brosimum gaudichaudii Tréc) in natura, proveniente do Cerrado Mineiro, a fim de trazer para a comunidade científica mais dados acerca da composição nutricional deste fruto, pois foi encontrado somente um único estudo, de Rocha, ${ }^{11}$ o qual analisou a composição centesimal (exceto fibras alimentares), parâmetros físico-químicos (sólidos solúveis, atividade antioxidante, $\mathrm{pH}$ ) e compostos bioativos (compostos fenólicos totais, flavonoides, antocianinas, $\beta$-caroteno, licopeno e vitamina C) em mama-cadela (chamada de bureré) proveniente do Cerrado Piauiense.

Dessa forma, não foram encontrados, ate o presente momento, dados de composição nutricional, compostos bioativos e características físico-químicas de mama-cadela proveniente do Cerrado Mineiro. Com base no exposto, ressalta-se a importância de trazer novos elementos de composição nutricional para frutos regionais (particularmente a mama-cadela), uma vez que um mesmo fruto pode ter diferenças em sua composição nutricional devido, principalmente, às diversificações de clima e solo em regiões do Brasil, sobretudo no Cerrado Mineiro e Piauiense. 


\section{Metodologia}

A mama-cadela (Brosimum gaudichaudii Tréc) in natura com casca e madura foi colhida na safra de 2014, que foi entre os meses de outubro e novembro, por um produtor local da cidade de UberlândiaMG. Em seguida, a mama-cadela foi transportada para o Laboratório de Bromatologia e Microbiologia de Alimentos da Universidade Federal de Uberlândia (UFU) e, posteriormente, armazenada sem processamentos e congelada a temperatura de $-18^{\circ} \mathrm{C}$. O experimento consistiu em: uma tomada de ensaio, uma unidade amostral (em torno de 3,5 quilos de mama-cadela), fruto obtido em um único local, e todas as análises foram feitas em triplicata. ${ }^{12}$ Para as análises, retirou-se apenas a semente, o restante foi utilizado.

A composição centesimal (umidade, cinzas, proteínas, fibras alimentares, carboidratos "disponíveis", lipídios e valor energético (em kcal e kJ), os parâmetros físico-químicos ( $\mathrm{pH}$, acidez total titulável e atividade antioxidante) e os compostos bioativos (vitamina C e fenólicos totais) da mama-cadela foram analisados de acordo com métodos propostos pela Association of Official Analytical Chemists ${ }^{13}$ e pelo Instituto Adolfo Lutz ${ }^{14}$ e descritos detalhadamente por Da Paz et al., ${ }^{15}$ Pacheco et al. ${ }^{12}$ e Silva et al., ${ }^{16}$ como se segue: a determinação de umidade foi baseada na perda de peso da amostra submetida a aquecimento em estufa a $105{ }^{\circ} \mathrm{C}$ por 24 horas. ${ }^{14}$ As cinzas foram determinadas pelo peso de material que restou após incineração em mufla a 550 ํㅡ por 6 a 8 horas. ${ }^{13,14}$ Para determinação da proteína, foi utilizado o método de micro-Kjeldhal ${ }^{13} \mathrm{e}$ para a estimativa do teor de proteínas, o teor de nitrogênio foi multiplicado pelo fator de conversão para vegetais $\left(\mathrm{N}\right.$ x 5,75) $\cdot{ }^{17} \mathrm{~A}$ fibra alimentar total foi estabelecida pelo método enzímico-gravimétrico. ${ }^{13} \mathrm{O}$ teor de carboidratos $(\mathrm{CHO})$ "disponíveis" foi definido por diferença: CHO "disponíveis" $(\%)=100 \%$ - (\%umidade + \%proteínas + \%lipídios + \%cinzas + \%fibras alimentares). ${ }^{17} \mathrm{O}$ teor de lipídios foi determinado pelo método de Goldfish. ${ }^{13}$ Os compostos fenólicos foram estipulados de acordo com o método de Singleton e Rossi ${ }^{18}$ e os seus resultados foram expressos em: mg de ácido gálico por $100 \mathrm{~g}$ de alimento na base úmida. A determinação de vitamina $\mathrm{C}$ foi feita pelo método de iodato de potássio. ${ }^{14} \mathrm{~A}$ atividade antioxidante foi avaliada segundo a técnica descrita por Brand-Williams (porcentagem de inibição do radical DPPH). ${ }^{19}$ As análises físico-químicas foram: potencial hidrogeniônico $(\mathrm{pH})^{13}$ e acidez total titulável. ${ }^{14}$ Para o cálculo energético, o valor de carboidratos “disponíveis" e de proteínas foi multiplicado por $4 \mathrm{kcal} / \mathrm{g}$ e o valor de lipídios, por $9 \mathrm{kcal} / \mathrm{g}$. O valor energético (em kcal) foi multiplicado por 4,184 para transformar em kJ. A média e o desvio-padrão da composição centesimal, compostos bioativos e dos parâmetros físico-químicos foram obtidos com o auxílio do software Microsoft Office Excel (2010).

\section{Resultados}

Os dados obtidos referentes à composição centesimal e ao valor energético de mama-cadela (Brosimum gaundichaudii Tréc) estão apresentados na Tabela 1. 
Tabela 1. Composição centesimal (g/100 g) e valor energético (kcal/100 g e kJ/100 g) da mamacadela (Brosimum gaudichaudii Tréc) in natura, na base úmida e na base seca. Uberlândia, Minas Gerais, 2014.

\begin{tabular}{lcc}
\hline \multicolumn{1}{c}{ Composição centesimal } & $\begin{array}{c}\text { Base Úmida } \\
\text { Média } \pm \text { Desvio-padrão }\end{array}$ & $\begin{array}{c}\text { Base Seca } \\
\text { Média } \pm \text { Desvio-padrão }\end{array}$ \\
\hline Umidade & $77,63 \pm 1,46$ & - \\
Proteínas & $1,63 \pm 0,05$ & $7,27 \pm 0,23$ \\
Lipídios & $0,60 \pm 0,02$ & $2,67 \pm 0,08$ \\
Cinzas & $0,82 \pm 0,01$ & $3,66 \pm 0,06$ \\
CHO “disponíveis” & & $59,66 \pm 9,70$ \\
(por diferença) & $13,35 \pm 2,17$ & \\
FAT & $5,11 \pm 1,80$ & $22,86 \pm 8,05$ \\
VET $^{1}$ & $65,21 \pm 8,34$ & $291,48 \pm 37,27$ \\
VET $^{2}$ & $272,82 \pm 34,88$ & $1219,57 \pm 155,93$ \\
\hline
\end{tabular}

Valores médios \pm desvio-padrão (DP); $n=3$ (triplicata de análise); FAT = fibras alimentares totais; VET $^{1}=$ valor energético total em $\mathrm{kcal} / 100 \mathrm{~g} ; \mathrm{VET}^{2}=$ valor energético total em $\mathrm{kJ} / 100 \mathrm{~g}$.

Os dados obtidos referentes aos parâmetros físico-químicos e compostos bioativos de mamacadela (Brosimum gaundichaudii Tréc) estão apresentados na Tabela 2.

Tabela 2. Parâmetros físico-químicos e compostos bioativos da mama-cadela (Brosimum gaudichaudii Tréc) in natura, na base úmida. Uberlândia, Minas Gerais, 2014.

\begin{tabular}{lc}
\multicolumn{1}{c}{$\begin{array}{c}\text { Parâmetros físico-químicos } \\
\text { e compostos bioativos }\end{array}$} & Média \pm desvio-padrão \\
\hline $\mathrm{pH}$ & $5,96 \pm 0,03$ \\
Acidez total titulável (\% ácido cítrico) & $5,85 \pm 0,18$ \\
Atividade antioxidante (\% de inibição do & $28,85 \pm 2,66$ \\
radical DPPH) & \\
Vitamina C (mg/100 g) & $14,92 \pm 1,68$ \\
Compostos fenólicos (mgEAG/100 g) & $46,47 \pm 0,73$ \\
\hline $\mathrm{n}=3$ (triplicata de análise).
\end{tabular}

$\mathrm{n}=3$ (triplicata de análise). 


\section{Discussão}

Com relação à composição centesimal (Tabela 1), o teor de umidade do presente estudo foi de $77,63 \%$, evidenciando que a mama-cadela apresenta alto teor de água em sua composição. Rocha, ${ }^{11}$ em um estudo também conduzido com mama-cadela do Cerrado Piauiense (chamado na região de bureré), encontrou 72,3\% de umidade, valor próximo ao da presente pesquisa. Da Paz et al. ${ }^{15}$ encontraram 52,4\% de umidade no pequi, que é também um fruto do Cerrado. No jenipapo, Pacheco et al. ${ }^{12}$ acharam $70 \%$. A umidade em frutos, de uma maneira geral, é bastante alta, e esse parâmetro pode ser um indicativo de baixa vida de prateleira, devido à alta atividade microbiológica que a água proporciona..$^{20,21}$

O teor de proteínas observado neste estudo foi de 1,63\%, valor abaixo do estudo de Rocha, ${ }^{11}$ que encontrou 2,2\%. Silva et al. ${ }^{22}$ analisaram diversos frutos do Cerrado, como araticum, cagaita, gabiroba e mangaba e encontraram, respectivamente, 1,22\%, 0,82\%, 0,50\% e 1,20\%. Comparando os valores proteicos desses frutos com a mama-cadela do presente estudo, percebeu-se que ela possui maior quantidade de proteínas.

O teor de lipídios do presente trabalho $(0,60 \%)$ foi duas vezes maior que o encontrado por Rocha $(0,30 \%) .{ }^{11}$ Silva et al. ${ }^{22}$ notaram quantidades parecidas em outros frutos do Cerrado, como: cagaita $(0,44 \%)$ e caju do cerrado (0,63\%). Pacheco et al. ${ }^{12}$ não encontraram lipídios em frutos de jenipapo.

As cinzas do presente estudo (0,82\%) foram abaixo do encontrado por Rocha, ${ }^{11}$ que obteve $1,3 \%$. As cinzas indicam a presença de minerais em um fruto, e seus teores podem variar de acordo com a qualidade dos solos. Vários fatores podem interferir na qualidade nutricional de solos: diferenças em relevo, altitude em relação ao nível do mar, período de chuvas e escassez da região, o ar, a qualidade da água, a própria vegetação (se é densa ou não), a presença de seres vivos (animais da região ou o próprio homem), entre outros fatores. ${ }^{23}$ Em comparação a outros frutos do Cerrado, Morzelle et al. ${ }^{24}$ obtiveram 0,84\%, 1,32\%, 2,31\%, em curriola, gabiroba e murici, respectivamente. Pacheco et al. ${ }^{12}$ encontraram 1,1\% de cinzas em jenipapo. Observa-se, neste caso, que as cinzas da mama-cadela estão abaixo dos valores de outros frutos, podendo indicar que apresenta poucos minerais na sua composição química.

Os carboidratos "disponíveis" da mama-cadela da presente pesquisa foram de 13,35\%. Rocha" encontrou 23,8\% de carboidratos totais (carboidratos "disponíveis" + fibras alimentares) na sua pesquisa, também na base úmida, pois os carboidratos totais foram calculados por diferença e sem descontar o valor das fibras alimentares. Se somarmos os valores de carboidratos "disponíveis" e fibras alimentares da presente pesquisa, obteremos o valor de 18,46\%, ficando abaixo do encontrado por Rocha. ${ }^{11}$ De acordo com outros estudos com frutos do Cerrado, é possível perceber algumas variações no teor de carboidratos "disponíveis", como no araticum $(12,78 \%)$, na cagaita $(3,08 \%)$, na macaúba $(35,06 \%)$, na gabiroba $(15,68 \%)$ e no pequi $(3,4 \%) \cdot{ }^{15,22,24}$ 
As fibras alimentares totais (FAT) da mama-cadela do presente estudo foram de $5,11 \%$ e não foram encontrados valores de fibras alimentares de mama-cadela em outras pesquisas. Em estudos com o jenipapo, ${ }^{12,21}$ foram observados $1,09 \%$ e 6,3\% de FAT. Em outra pesquisa, Abreu et al..$^{25}$ obtiveram 5,89\% e 6,81\% de FAT em amostras de pitaia branca e vermelha, respectivamente. $\mathrm{O}$ valor energético em $100 \mathrm{~g}$ de parte comestível de mama-cadela foi de 65,21 kcal (272,82 kJ), o qual corresponde a 3,26\% das necessidades diárias de um indivíduo adulto saudável (2.000 kcal por dia). ${ }^{26}$

Com relação aos parâmetros físico-químicos (Tabela 2), o valor de $\mathrm{pH}(5,96)$ encontrado no presente estudo foi maior que o descrito por Rocha $(5,7) .{ }^{11}$ Há frutos do Cerrado com pH mais ácido que o da mama-cadela, como, por exemplo, a cagaita $(2,97)$ e a gabiroba $(3,97) .{ }^{27}$ No presente estudo, a acidez total titulável foi de $5,85 \%$. Rocha ${ }^{11}$ obteve um valor maior $(7,1 \%)$. Perfeito et al..$^{28}$ encontraram $0,84 \%$ na mangaba. Da Paz et al., ${ }^{15} 0,7 \%$ no pequi. É possível observar que a acidez da mama-cadela foi superior à encontrada em outros frutos do Cerrado brasileiro.

A atividade antioxidante da mama-cadela do presente estudo foi de $28,85 \%$. Pacheco et al. ${ }^{12}$ demonstraram que a atividade antioxidante no jenipapo foi de $70,2 \%$. Da Paz et al. ${ }^{15}$ obtiveram $13,7 \%$ no pequi. Vale elucidar que os valores discrepantes de atividade antioxidante entre os diversos frutos do Cerrado demonstram a diversidade deste bioma.

Os compostos fenólicos da mama-cadela do presente estudo (46,47 mgEAG/100 g) foram superiores ao estudo de Rocha ${ }^{11}$ (20,73 $\left.\mathrm{mgEAG} / 100 \mathrm{~g}\right)$. Comparando a mama-cadela do presente estudo com outros frutos do Cerrado, Abreu et al. ${ }^{25}$ analisaram pitaias branca e vermelha e encontraram 116,14 mgEAG/100 g e 77,22 mgEAG/100 g, respectivamente. Da Paz et al. ${ }^{15}$ encontraram no pequi 531,5 mgEAG/100 g. A vitamina C da mama-cadela do presente trabalho foi de 14,92 mg/100 g, valor muito abaixo do estudo de Rocha ${ }^{11}(86,5 \mathrm{mg} / 100 \mathrm{~g})$. Rocha ${ }^{29}$ verificou que a vitamina $\mathrm{C}$ estava presente em boas quantidades no cajuí (500 mg/100 g), jatobá (330,4 $\mathrm{mg} / 100 \mathrm{~g})$ e na macaúba $(185,1 \mathrm{mg} / 100 \mathrm{~g})$. Essas diferenças substanciais dos compostos fenólicos e da vitamina $\mathrm{C}$ do presente estudo com a literatura podem ser decorrentes das diferenças metodológicas (por exemplo: solventes utilizados), da origem da amostra (o Brasil apresenta dimensões continentais e há diversidades de solo e clima entre as regiões) e da forma e do tempo de armazenamento do fruto. ${ }^{23}$

A mama-cadela do presente estudo obteve destaques quanto aos teores de carboidratos totais (18,46\% na base úmida), particularmente as fibras alimentares, e de compostos fenólicos. O Cerrado brasileiro pode ser considerado uma fonte de alimentos à população que ali habita, principalmente fornecendo diversos frutos nativos comestíveis. A mama-cadela é um fruto bastante conhecido na região do Cerrado, mas que carece de pesquisas quanto as suas características nutricionais e funcionais. 
O incentivo ao consumo de mama-cadela vai ao encontro das políticas públicas do governo brasileiro, que apoia a preservação do Cerrado e incentiva a utilização de alimentos regionais, garantindo, dessa forma, a segurança alimentar e nutricional da população. ${ }^{30}$ É evidente a necessidade de pesquisas que aprofundem os aspectos nutricionais e funcionais da mama-cadela, visto que foi encontrado apenas um estudo com mama-cadela proveniente do Cerrado Piauiense, ${ }^{11}$ ao passo que o presente estudo foi feito com o fruto do Cerrado Mineiro.

\section{Conclusão}

Com relação à composição centesimal da mama-cadela, os destaques foram para as fibras alimentares (cerca de 5\%) e os carboidratos "disponíveis" (aproximadamente 13\%), totalizando quase $18 \%$ de carboidratos totais. Ao que se refere aos compostos bioativos, os destaques foram para os compostos fenólicos. No geral, a mama-cadela pode ser considerada um novo alvo de estudos científicos, ficando clara a evidência de que é necessário o aprofundamento das suas características nutricionais e funcionais.

\section{Colaboradores}

Todos os autores contribuíram na concepção e desenho do estudo, análise de dados e redação final. Conflito de Interesses: Os autores declaram não haver conflito de interesses.

\section{Referências}

1. Malta, LG. Avaliação biológica de frutas do Cerrado brasileiro: Guapeva, Gabiroba e Murici. [Tese]. [Campinas]: Universidade Estadual de Campinas. Faculdade de Engenharia de Alimentos; 2011.

2. Brasil. Ministério do Meio Ambiente. O bioma cerrado [Internet]. [acesso em: 05 abr. 2017]. Disponível em: http://www.mma.gov.br/biomas/cerrado

3. Santos MAD, Barbieri AF, Guedes GR, Machado CJ, Carvalho JAM. Dinâmica demográfica e uso da terra no cerrado brasileiro: reflexões a partir da experiência do Padap. Rev Econ Sociol Rural 2012; 50(2):319-331.

4. Brasil. Ministério do Meio Ambiente. Programa de Cooperação entre Brasil, Reino Unido e Banco Mundial "Redução do desmatamento e das queimadas no cerrado brasileiro". Marco de gestão social e ambiental. Brasília: MMA; 2014.

5. Souza VR, Pereira PAP, Queiroz F, Borges SV, Carneiro JDS. Determination of bioactive compounds, antioxidant activity and chemical composition of Cerrado Brazilian fruits. Food Chemistry 2012; 134(1)381-386. 
6. Ramos MIL, Ramos Filho MM, Hiane PA, Braga Neto JA, Siqueira EMA. Qualidade nutricional da polpa de Bocaiúva Acrocomia aculeata (Jacq.) Lodd. Ciênc Tecnol Aliment. 2008; 28:90-94.

7. Roesler R, Malta LG, Carrasco LC, Holanda RB, Sousa CAS, Pastore GM. Atividade antioxidante de frutas do cerrado. Ciênc Tecnol Aliment. 2007; 27(1):56-60.

8. Faria RAPG, Silva AN, Albuquerque MCF, Coelho MFB. Características biométricas e emergência de plântulas de Brosimum gaudichaudii Tréc. oriundas de diferentes procedências do cerrado matogrossense. Rev Bras Pl Med. 2009; 11(4):414-421.

9. Silva DB, Vieira RF, Cordeiro MCT, Pereira EBC, Pereira AV. Propagação vegetativa de Brosimum gaudichaudii Tréc. (mama-cadela) por estacas de raízes. Rev Bras Pl Med. 2011; 13(2):151-156.

10. Jacomassi E, Moscheta IS, Machado SR. Morfoanatomia e histoquímica de órgãos reprodutivos de Brosimum gaudichaudii (Moraceae). Rev Brasil Bot. 2010; 33(1):115-129.

11. Rocha MS. Compostos bioativos e atividade antioxidante (in vitro) de frutos do cerrado piauiense. [Dissertação]. [Teresina]: Universidade Federal do Piauí, Programa de Pós-Graduação em Alimentos e Nutrição; 2011.

12. Pacheco P, Paz JG, Silva CA, Pascoal GB. Composição centesimal, compostos bioativos e parâmetros físico-químicos do jenipapo (genipa americana L.) in natura. Demetra 2015; 9(4):1041-1054,.

13. Association of Official Analytical Chemists. Official methods of analysis. 16. ed. Washington: AOAC; 1997.

14. Instituto Adolfo Lutz. Normas analíticas do Instituto Adolfo Lutz: métodos físico-químicos para análise de alimentos. São Paulo: Instituto Adolfo Lutz; 2008. 1020 p.

15. Paz JG, Pacheco P, Silva CO, Pascoal GB. Análise da Composição nutricional e de parâmetros físicoquímicos do Pequi (Caryocar Brasiliense Camb) in natura. Rev Científica Linkania Master 2014; 1(8):74-159.

16. Silva ACB, Schuquel LCS, Da Silva CO, Pascoal GB. Qualidade nutricional e físico-química em cenoura (Daucus Carota L.) in natura e minimamente processada. Demetra 2016; 11(2):355-367.

17. Brasil. Agência Nacional de Vigilância Sanitária. Resolução RDC n. 360 de 23 de dezembro de 2003: Regulamento técnico sobre rotulagem nutricional de alimentos embalados. Diário Oficial da União 26 dez. 2003.

18. Singleton VL, Rossi JA. Colorimetry of total phenolics with phosphomolybdic-phosphotungstic acid reagents. Am J Enol Vitic 1965; 16(3):144-158.

19. Brand-Williams W, Cuvelier ME, Berset C. Use of a free radical method to evaluate antioxidant activity. LWT-Food Science and Technology 1995; 28(1)25-30.

20. Hiane PA, Ramos MIL, Ramos Filho MM, Pereira JG. Composição centesimal e perfil de ácidos graxos de alguns frutos nativos do Estado de Mato Grosso do Sul. Centro de Pesquisa de Processamento de Alimentos 1992; 10(1):35-42.

21. Hamacek FR, Moreira AVB, Martino HSD, Ribeiro SMR, Pinheiro-Sant'Ana HM. Valor nutricional, caracterização física e físico-química de jenipapo (Genipa americana L.) do cerrado de Minas Gerais. Alim. Nutr. 2013; 24(1):73-77. 
22. Silva MR, Lacerda DBCL, Santos GG, Martins DMO. Caracterização química de frutos nativos do cerrado. Ciênc Rural 2008; 38(6):1790-1793.

23. Gomes RLR, Silva MC, Costa FR, Lima Junior AF, Oliveira IP, Silva DB. Propriedades físicas e teor de matéria orgânica do solo sob diferentes coberturas vegetais. Revista Faculdade Montes Belos 2015; 8(1):72-139.

24. Morzelle MC, Bachiega P, Souza EC, Vilas Boas EVB, Lamounier ML. Chemical And Physical Charecteriztion Of Fruits From Cerrado: Curriola, Gabiroba And Murici. Rev Bras Frutic. 2015; 37(1):96-103.

25. Abreu WC, Lopes CO, Pinto KM, Oliveira LA, Carvalho GBM, Barcelo MFP. Características físico-químicas e atividade antioxidante total de pitaias vermelha e branca. Rev. Inst Adolfo Lutz 2012; 71(4)656-661.

26. Brasil. Ministério da Saúde. Secretaria de Atenção à Saúde. Guia alimentar para a população brasileira: promovendo a alimentação saudável. Brasília: Ministério da Saúde; 2008.

27. Silva AML, Martins BA, Deus TN. Avaliação do teor de ácido ascórbico em frutos do Cerrado durante o amadurecimento e congelamento. Estudos 2009; 36(6):1159-1169.

28. Perfeito DGA, Carvalho N, Lopes MCM, Schmidt FL. Caracterização de frutos de mangabas (Hancornia speciosa Gomes) e estudo de processos de extração da polpa. Revista Agricultura Neotropical 2015; 2(3):1-7.

29. Rocha MS,Figueiredo RW, Araújo MAM, Moreira-Araújo RSR. Caracterização físico-química e atividade antioxidante (in vitro) de frutos do cerrado piauiense. Rev Bras Frutic. 2013; 35(4):933-941.

30. Brasil. Ministério da Saúde. Secretaria de Atenção à Saúde. Departamento de Atenção Básica. Alimentos regionais brasileiros. Brasília: Ministério da Saúde; 2015. 484 p. 